\begin{abstract}
UNIVERSIDADE TECNOLÓGICA FEDERAL DO PARANÁ DEPARTAMENTO ACADÉMICO DE MECÂNICA

CURSO DE ESPECIALIZAÇÃO EM ENGENHARIA DE PRODUÇÃO
\end{abstract}

RAFAEL DAROS

ANÁLISE DE VIABILIDADE ECONÔMICA DA AQUISIÇÃO DE UM EQUIPAMENTO UTILIZANDO A METODOLOGIA MULTI-ÍNDICE AMPLIADA

MONOGRAFIA DE ESPECIALIZAÇÃO

PATO BRANCO - PR 
RAFAEL DAROS

\section{ANÁLISE DE VIABILIDADE ECONÔMICA DA AQUISIÇÃO DE UM EQUIPAMENTO UTILIZANDO A METODOLOGIA MULTI-ÍNDICE} AMPLIADA

Monografia de Especialização apresentada ao Departamento Acadêmico de Mecânica, da Universidade Tecnológica Federal do Paraná como requisito parcial para obtenção do título de "Especialista em Engenharia de Produção". Orientador: Prof. Dr. José Donizetti De Lima

PATO BRANCO - PR 


\section{TERMO DE APROVAÇÃO}

ANÁLISE DE VIABILIDADE ECONÔMICA DA AQUISIÇÃO DE UM EQUIPAMENTO UTILIZANDO A METODOLOGIA MULTI-ÍNDICE AMPLIADA

por

\section{RAFAEL DAROS}

Esta Monografia foi apresentada em vinte e quatro de março de 2017 como requisito parcial para a obtenção do título de Especialista em Engenharia de Produção. $\mathrm{O}(\mathrm{a})$ candidato(a) foi arguido(a) pela Banca Examinadora composta pelos professores abaixo assinados. Após deliberação, a Banca Examinadora considerou o trabalho aprovado.

\begin{tabular}{c}
\hline José Donizetti de Lima \\
Prof.(a) Orientador(a) \\
\hline Marcelo Gonçalves Trentin \\
Membro titular \\
\hline Gilson Adamczuk Oliveira \\
Membro titular
\end{tabular}




\section{AGRADECIMENTOS}

Gostaria de Agradecer a Deus por me permitir finalizar este trabalho. A minha Família e amigos pelo apoio na fase mais difícil de minha vida.

Ao Dr. Oncologista Gilmar Juliani Biscaia, pelas inúmeras consultas, cirurgia e até então o tratamento do Melanoma a qual recebi o diagnostico ao final das disciplinas.

Também ao meu orientador pela dedicação e auxilio.

A vida sem luta é um mar morto no centro do organismo universal. 


\title{
RESUMO
}

Esta pesquisa tem por objetivo analisar a viabilidade econômica da aquisição de um equipamento para o acabamento de peças em uma fundição localizada no sudoeste do Paraná. A pesquisa é caracterizada como um estudo de caso, aplicando a metodologia Multi-índice Ampliada (MMIA) proposta por Lima et al. (2016), comparando os resultados das simulações com e sem impostos, como ferramenta de análise por meio do aplicativo $\$ \Lambda \bigvee € \pi$. Como resultados encontrados, destacamse: (i) retorno esperado, considerando a redução de custos de processo, o qual apresenta grau baixo-médio desconsiderando os impostos $(27,37 \%)$ e retorno baixo $(14,81 \%)$ considerando os impostos no lucro líquido; (ii) riscos estimados, com nível médio para o Payback/N sem impostos (59,17\%), com impostos apresenta riscos de nível alto (74,17\%) e TMA/TIR $57,46 \%$ e $72,23 \%$ sem e com impostos respectivamente; e (iii) limites de elasticidade, com média-alta tolerância às variações da TMA sem impostos e baixa-média variação da TMA considerando os impostos sobre o lucro líquido. Esses resultados apontam para a viabilidade econômica, mostrando que é recomendável a aquisição do equipamento analisado.

Palavras-chave: Análise econômica, Indicadores Econômicos, MMIA.

\begin{abstract}
This research aims to analyze the economic viability of the acquisition of an equipment for the finishing of parts in a foundry located in the southwest of Paraná. The research is characterized as a case study, applying the Multi Expanded Index (MMIA) methodology proposed by Lima et al. (2016), comparing the results of the simulations with and without taxes, as a tool of analysis through the application $\$ \Lambda \vee € \pi$. The following results were obtained: (i) expected return, considering the reduction of process costs, which presents low to medium grade, disregarding taxes $(27.37 \%)$ and low return (14.81\%) considering taxes In net income; $(59.17 \%)$, with high taxes (74.17\%) and TMA / IRR $57.46 \%$ and $72.23 \%$ without taxes. And with taxes respectively; And (iii) elasticity limits, with medium-high tolerance to the variations of the TMA without taxes and low-average variation of the TMA considering the taxes on the net profit. These results point to the economic viability, showing that it is advisable to purchase the analyzed equipment.
\end{abstract}

Keywords: Economic Analysis, Economic Indicators, MMIA. 


\section{SUMÁRIO}

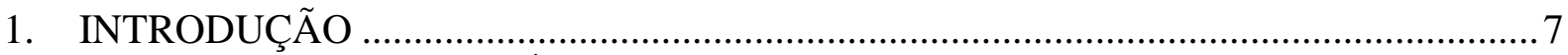

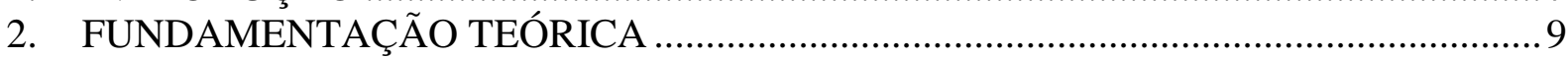

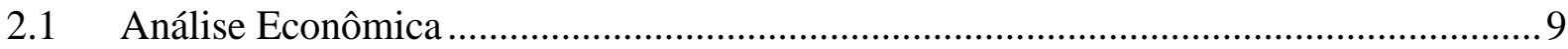

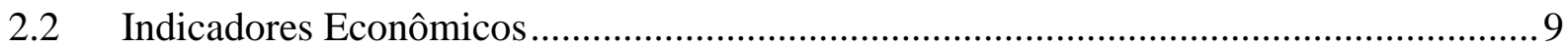

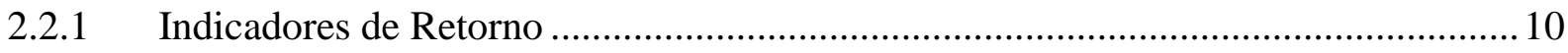

2.2.2 Indicadores de Risco ................................................................................ 11

2.3 Metodologia Multi Índice Ampliada (MMIA) ……………....................................... 12

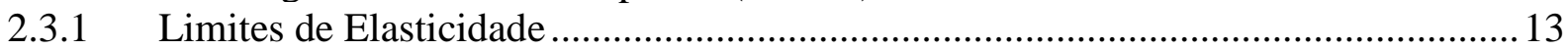

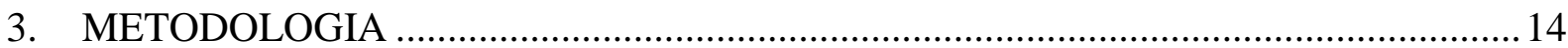

4. APRESENTAÇÃO E DISCUSSÃO DOS RESULTADOS ...............................................

4.1 Caracterização da Empresa e do Projeto de Investimento.................................................. 17

4.2 Análise Econômica do Projeto de Investimento Proposto................................................... 17

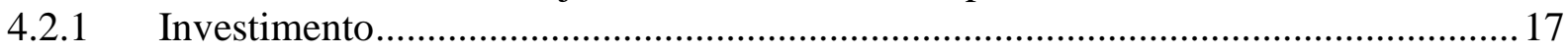

4.3 Custos de Produção dos Processos ........................................................................... 18

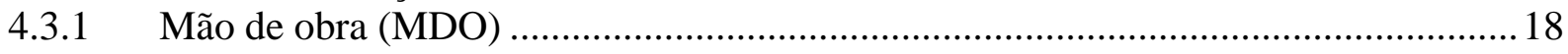

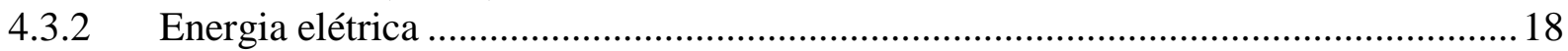

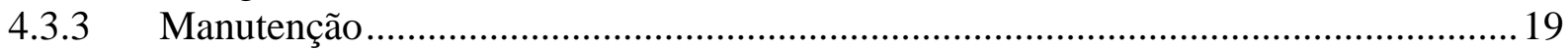

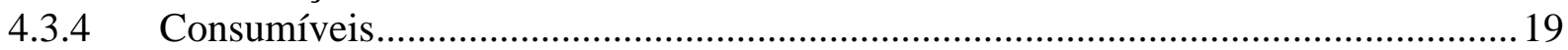

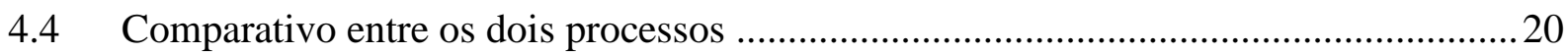

4.5 Estudo de Viabilidade Econômica do Projeto de Investimento ………………………....22

4.5.1 Inserção dos dados no Aplicativo Web $\$ \Lambda \mathrm{V} € \pi$ sem impostos .....................................2 20

4.5.2 Inserção dos dados no Aplicativo Web $\$ \Lambda \mathrm{V} € \pi$ com impostos......................................2

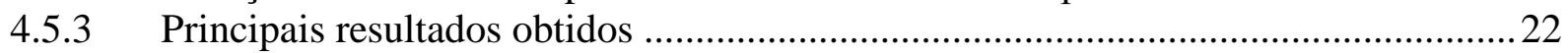

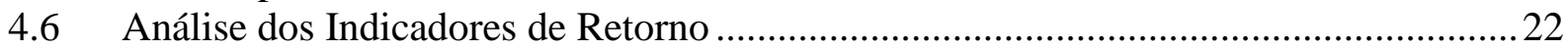

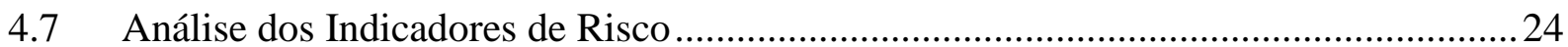

4.8 Análise dos Limites de Elasticidade e Comparativo entre Retorno e Riscos ................25

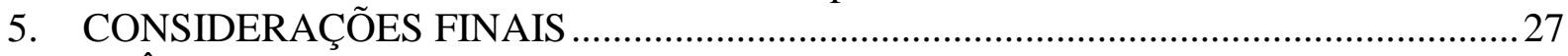

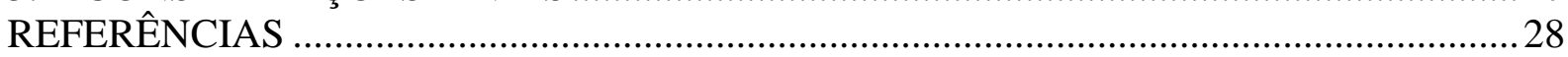




\section{INTRODUÇÃO}

A elevada complexidade do processo administrativo levam os gestores a buscarem alternativas para superar os desafios encontrados no dia a dia. $O$ planejamento financeiro determina as diretrizes de mudança em uma empresa e servem para estabelecer metas, motivar a organização e avaliar seu desempenho, bem como em um mundo de incertezas, a empresa deve estar preparada para mudança de condições e surpresas (ROSA, 2010).

Inicialmente deve ser realizada a verificação da viabilidade técnica de um projeto de investimento (PI) no qual é determinada pela capacidade de produção, além de considerações referentes à seleção entre os diversos processos produtivos, a engenharia de projeto e ao arranjo físico dos equipamentos na fabrica. $A$ viabilidade técnica tem foco nos processos produtivos e mudanças tecnológicas do investimento, por isso, faz-se necessário a avaliação da necessidade produtiva, bem como a análise das projeções de venda (STRACHOSKI, 2011).

Ferro et al. (2016) utilizaram a Metodologia Multi-Índice Ampliada, para análise de viabilidade econômica da aquisição de uma linha de pintura, enquanto que ARTUZO et al. (2016), utilizou a mesma metodologia para realizar a análise de viabilidade econômica da alteração de um processo de moldagem de chapas de fogão á lenha.

Segundo Ferro et al. (2016) existem indicadores econômicos para análise de projetos de investimento e podem ser divididos em dois grupos, sendo o primeiro, os indicadores associados à rentabilidade do projeto, sendo eles: o Valor Presente Líquido (VPL), Valor Presente Líquido Anualizado (VPLA), Índice de Benefício Custo (IBC), Retorno Adicional Sobre o Investimento (ROIA) e o índice ROIA/TMA, sendo TMA, a Taxa Mínima de Atratividade; e o segundo relacionado a indicadores referentes aos riscos do projeto, sendo eles: Taxa Interna de Retorno (TIR), o Período de Recuperação do Investimento (Payback) e os índices TMA/TIR e Payback/N, em que $\mathrm{N}$ é o horizonte de planejamento.

A previsão do fluxo de caixa e a utilização de indicadores econômicos, bem como os limites de elasticidade para possíveis variações na analise utilizadas na Metodologia Multi-Índice Ampliada (MMIA), é um diferencial para auxiliar na tomada de decisão para uma possível aquisição de um projeto de investimento (PI) (LIMA et al., 2015). 
Em decorrência dos benefícios da aplicação da metodologia MMIA, este estudo tem por objetivo realizar uma análise de viabilidade financeira de um equipamento para o setor de acabamento em uma fundição localizada na região sudoeste do Paraná, com auxilio da ferramenta $\$ \wedge \bigvee € \pi$. 


\section{FUNDAMENTAÇÃO TEÓRICA}

\subsection{Análise Econômica}

Segundo Rozenfeld et al. (2006), o estudo de viabilidade econômica muitas vezes esta vinculado a elaboração de um plano de negócios e geralmente é aplicado em empresas que apresentam uma estrutura consolidada de funcionamento que se interessa em investir em novos ramos de mercado, melhorias de infraestrutura ou ampliação da área de atuação, ao se estabelecer um $\mathrm{PI}$, o mesmo requer recursos humanos, financeiros, equipamentos e de infraestrutura. A análise da viabilidade econômica de um PI envolve o estudo de fases que abordam o conhecimento sobre o mercado que pretende atuar e a previsão de faturamento, com essas informações é possível efetuar o cálculo dos indicadores que avaliarão a sua viabilidade econômica.

Para elaborar a análise econômica e financeira de um PI, somente deve se considerar fatores conversíveis em dinheiro então, caso um investimento tenha repercussões que não sejam ponderáveis, tais como: redução do impacto ambiental, nível de emprego, vendas futuras e boa vontade de clientes e fornecedores, em geral, são critérios imponderáveis (CASAROTTO FILHO E KOPITTKE, 2010).

Tendo valores conversíveis em dinheiro, são gerados indicadores econômicos que auxiliarão o processo de tomada de decisão. Os analistas devem utilizar técnicas que considerem o valor de dinheiro ao longo de uma escala de tempo para reconhecer oportunidades de obter resultados positivos quando avaliando as séries de fluxos de caixa esperados associados às alternativas, também deve ser determinada a taxa de juros a ser empregada como parâmetro para avaliação econômica (NOGUEIRA, 2009).

Para Casarotto Filho e Kopittke (2010), a TMA pode ser igual à taxa de juros da empresa no mercado, essa definição é adotada no restante desse trabalho.

\subsection{Indicadores Econômicos}

Souza e Clemente (2008), afirmam que para realizar a análise da viabilidade econômica e financeira de um Projeto de Investimento (PI) pode ser utilizada a Metodologia Multi-índice (MMI) onde a metodologia, na qual vários indicadores 
econômicos, auxiliam o processo decisório quanto à aceitação ou rejeição do projeto. Os indicadores são classificados em relação ao retorno, aos riscos e para melhorar a percepção, podem ser analisados por meio de sensibilidade (LIMA et al., 2015).

\subsubsection{Indicadores de Retorno}

\section{- Valor Presente (VP)}

O valor presente (VP) ou valor atual (VA) representa o valor hoje de um fluxo ou de uma série futura de fluxos de caixa (BREALEY E MYERS, 1992). Para atualizar esses fluxos, é utilizada a taxa mínima de atratividade (TMA), que Kassai et al. (2000), define como:

Entende-se por taxa mínima de atratividade (TMA) a taxa mínima a ser alcançada em determinado projeto; caso contrário, o mesmo deve ser rejeitado. É, também, a taxa utilizada para descontar os fluxos de caixa quando se usa o método de valor presente líquido (VPL) e o parâmetro de comparação para a TIR. É o rendimento mínimo de uma segunda melhor alternativa do mercado.

\section{- Valor Presente Líquido (VPL)}

O Valor Presente Líquido (VPL) é o resultado do acumulo de todos os valores de um fluxo de caixa (FC), descontados para o presente, utilizando como taxa de desconto a TMA. Apresenta em valores monetários atual, a diferença entre os recebimentos e os pagamentos de todo o projeto (LIMA et al., 2013; LIMA et al., 2015), também pode ser descrito como valor presente dos fluxos futuros de caixa estimados para o projeto em análise. Se positivo, representa o ganho gerado ao longo da vida útil do projeto a valor presente pela TMA (HARZER et al., 2012).

\section{- Valor Presente Liquido Anualizado (VPLA)}

O Valor Presente Liquido Anualizado (VPLA) é uma derivação do VPL tradicional que o torna mais compreensível, pois o VPLA transforma o valor do VPL em uma série uniforme periódica equivalente onde representa o ganho gerado por unidade de tempo (HARZER et al., 2012). 
- Índice Beneficio Custo (IBC)

O índice de beneficio custo (IBC) é uma relação entre o VP e o investimento inicial e pode ser analisado como o retorno gerado por unidade monetária investida no projeto, ao final de sua vida útil (SOUZA e CLEMENTE, 2008).

\section{- Retorno Adicional sobre o Investimento (ROIA)}

O Retorno Adicional Sobre Investimento (ROIA) demonstra a melhor avaliação de rentabilidade, já eliminado o efeito da TMA, do projeto de investimento em análise. É comparado ao percentual do Valor Econômico Agregado (EVA Economic Value Added) (ROSOTO et al., 2012).

\subsubsection{Indicadores de Risco}

\section{- Taxa Interna de Retorno (TIR)}

Segundo Casarotto Filho e Kopittke (2010), o método da TIR exige o cálculo da taxa que zera o VPL dos fluxos de caixas (FCs) das alternativas. Esse indicador tem o objetivo de fazer com que os FCs, tanto de entrada (receitas) como de saída (custos), sejam iguais, ou seja, possuem a mesma taxa de desconto.

\section{- Período de Recuperação do Investimento (Payback)}

Este indicador de risco determina o número de períodos necessários para recuperar o investimento. Todos os valores dos fluxos de caixa do projeto são trazidos a valor presente e, quando a soma desses valores periódicos iguala ou supera o capital investido, tem-se o payback do investimento. $\mathrm{Na}$ análise tradicional de investimentos, o investidor, ao analisar estritamente sob a ótica do Payback, estipula o período máximo aceito de retorno do investimento e confronta com o valor calculado, caso este resultar em um número inferior, indica que o projeto deve ser aceito, caso superior, o projeto deve ser rejeitado (SOUZA e CLEMENTE, 2008). 


\subsection{Metodologia Multi Índice Ampliada (MMIA)}

A Metodologia Multi-Índice Ampliada (MMIA) é uma das mais recentes formas de se analisar um projeto de investimento (LIMA et al., 2016).

$\mathrm{Na}$ metodologia Multi-Índice, com exceção do ROIA, os demais indicadores de retorno são os mesmos encontrados na literatura clássica de análise de investimentos. Porém, um único indicador de forma isolada não suporta a decisão a ser tomada. Ao contrário, ao encontrar um VPL positivo, por exemplo, apenas significa que o projeto merece atenção e a análise continua com o cálculo dos demais indicadores de retorno e de medidas de risco. O uso conjunto dos indicadores produz informações mais consistentes do que seu uso isolado e se caracteriza pela avaliação dos riscos e do seu confronto com a expectativa de retorno (SOUZA e CLEMENTE, 2009).

Segundo Lima et al. (2015) e Lima (2016), para aplicar a MMIA em ativos fixos é necessário calcular indicadores de riscos e retorno associados ao PI. Além disso, deve fazer uma Análise de Sensibilidade (AS) sobre a TMA, os custos e as receitas para validar a manutenção da viabilidade econômica do $\mathrm{Pl}$. Esses índices para a AS, sua fórmula e a interpretação usual são apresentados na Figura 1.

\begin{tabular}{||c||l||}
\hline \multicolumn{1}{|c||}{ ÍNDICE } & \multicolumn{1}{c||}{ INTERPRETAÇÃO USUAL } \\
\hline \hline$\Delta \% \mathrm{TMA}_{\operatorname{máx} \uparrow}=\frac{T I R}{T M A}-1$ & $\begin{array}{l}\text { Aumento máximo admitido à TMA utilizada antes de inviabilizar } \\
\text { o PI em análise. Os demais parâmetros (Custos e Receitas, por } \\
\text { exemplo) são mantidos constantes. }\end{array}$ \\
\hline \hline$\Delta \% \mathrm{C}_{\max \uparrow}=I B C-1$ & $\begin{array}{l}\text { Aumento máximo nos Custos estimados antes de inviabilizar o PI } \\
\text { em avaliação. Os demais parâmetros (TMA e Receitas, por } \\
\text { exemplo) são mantidos constantes. }\end{array}$ \\
\hline \hline$\Delta \% \mathrm{R}_{\operatorname{máx}}=1-\frac{1}{I B C}$ & $\begin{array}{l}\text { Redução máxima nas Receitas esperadas antes de inviabilizar o PI } \\
\text { em estudo. Os demais parâmetros (TMA e Custos, por exemplo) } \\
\text { são mantidos constantes. }\end{array}$ \\
\hline
\end{tabular}

Fonte: Lima et al. (2015) e Lima (2016).

Figura 1 - Índices de Análise de Sensibilidade

Para Buarque (1989), o estudo da sensibilidade é muito importante quando a taxa de rentabilidade dos projetos não é elevada. A análise da sensibilidade informa aos responsáveis pelo projeto qual é o comportamento da rentabilidade. Além disso, ao comparar essa rentabilidade com outras alternativas, pode-se determinar se o projeto em estudo representa uma decisão coerente para o investimento.

De acordo com Souza e Clemente (2008), a ideia básica da análise de sensibilidade é a de verificar a sensibilidade da variação do VPL em função da 
alteração de um dos componentes do Fluxo de Caixa (FC). Assim, os parâmetros que provocarem maior variação do VPL serão classificados como sensíveis ou críticos.

\subsubsection{Limites de Elasticidade}

Lima et al. (2015) e Lima (2016) ampliaram os Indicadores de Viabilidade de Projetos de Investimentos (IVEPIs) da Metodologia Multi- índice (MMI) proposta por Souza e Clemente (2008). A expansão da MMI, denominada MMIA, refere-se à incorporação de Limites de Elasticidade (LEs) e Valores-Limite (VLs) para melhorar a percepção dos riscos. 


\section{METODOLOGIA}

Este trabalho, com relação à análise dos dados, classifica-se como uma pesquisa quantitativa e com relação à natureza da pesquisa, é tratado como um estudo de caso. O levantamento dos dados referentes aos custos para aquisição e instalação do equipamento, é determinado através de um orçamento realizado junto a um fabricante de equipamentos para fundições. As variações de custos do processo são estimadas com base nas informações de produtividade garantidas pelo fabricante do equipamento, bem como pela quantidade de operadores, energia elétrica consumida, custo de consumíveis dentre outros.

A TMA utilizada é de $1 \%$ ao mês, sendo esse o valor que a empresa tem definido para realização de projetos de investimento.

O recurso financeiro necessário para execução do projeto provém de recursos próprios.

Embora a aquisição do novo equipamento automatize uma etapa do processo de acabamento da fundição e reduza esforços físicos e riscos ergonômicos aos operadores, esse indicador não será mensurado durante a análise.

Para verificação da viabilidade econômica, as receitas serão mantidas constantes, mas servirão de base para determinar a diferença entre os custos do projeto atual com o futuro e utiliza-se da Metodologia Multi-Índice (MMI) proposta por Souza e Clemente (2008). Além disso, é utilizada a MMI Ampliada (MMIA) proposta por Lima et al. (2015) e ampliada por Lima (2016), conforme destaca a Figura 2. 


\begin{tabular}{|c|c|c|}
\hline DIMENSĀO & INDICADOR e FÓRMULA & INPUT E RESULTADO ESPERADO \\
\hline \multirow{7}{*}{ RETORNO } & $V P=\sum_{j=1}^{N} \frac{F C_{j}}{(1+T M A)^{\prime}}$ & TMA, N, FC,$F_{3}$ e N. VP $\geq\left|F C_{0}\right|$ \\
\hline & $V P L=-\left|F C_{0}\right|+V P$ & $\mathrm{VP}$ e $\mathrm{FC}_{0}$. VPL $\geq 0$ \\
\hline & $V P L A=\frac{V P L \times\left[T M A \times(1+T M A)^{\mathrm{v}}\right]}{\left[(1+T M A)^{x}-1\right]}$ & VPL, TMA e N. VPLA $\geq 0$. \\
\hline & $I B C=\frac{V P}{\left|F C_{\mathrm{a}}\right|}$ ou $I B C=\frac{V P(R)}{V P(C)}$ & VP E FC $C_{0}$ ou VP(R) e VP(C). IBC $\geq I$ \\
\hline & $R O I A=\sqrt[N]{I B C}-1$ & IBC e N. ROIA $\geq 0$ \\
\hline & Indice $R O I A / T M A=\frac{R O L A}{T M A}$ & TMA e ROIA. Índice ROIA/TMA $\mathbf{z} 0$. \\
\hline & $R O I=(1+T M A) \times(1+R O L A)-1$ & TMA e ROIA. ROIZ TMA. \\
\hline \multirow{4}{*}{ RISCOS } & $-\left|F C_{\mathrm{o}}\right|+\sum_{j=1}^{N} \frac{F C_{j}}{(1+T I R)^{\prime}}=0$ & $\mathrm{~N}, \mathrm{FC}_{0}$ e $\mathrm{FC}_{\mathrm{j}}$. TIR $\geq$ TMA. \\
\hline & Payback $=$ Minimo $\{j\}$ tal que $: \sum_{j=1}^{N} \frac{F C_{j}}{(1+T M A)^{\prime}} \geq\left|F C_{0}\right|$ & $\mathrm{N}, \mathrm{TMA}, \mathrm{FC}_{0}$ e $\mathrm{FC}_{\mathrm{j}}$ Payback $\leq \mathrm{N}$. \\
\hline & Indice $T M A / T I R=\frac{T M A}{T I R}$ & TMA e TIR. Índice TMA/TIR $\leq 100 \%$ \\
\hline & Indice Payback/ $N=\frac{\text { Payback }}{N}$ & Payback e N. Índice Payback/N $\leq 100 \%$. \\
\hline \multirow{5}{*}{ SENSIBILIDADE } & $\Delta \% \mathrm{TMA}_{\operatorname{mat}}=\gamma=\frac{T I R}{T M A}-1$ & TIR e TMA. $\boldsymbol{\gamma}>\mathbf{0}$ \\
\hline & $\Delta \% C_{\operatorname{mix}}=\varphi=I B C-1$ & IBC. $\varphi>0$. \\
\hline & $\Delta \% R_{\max }=\lambda=1-\frac{1}{I B C}$ & IBC. $\boldsymbol{\lambda}>0$. \\
\hline & $\Delta \%\left(C_{\text {mat }} \cap R_{\text {axiv }}\right)=\alpha=\frac{\lambda \cdot \varphi}{\lambda+\varphi}$ & $\lambda$ e $\varphi . \alpha>0$ \\
\hline & $\Delta \%\left(T M M_{\operatorname{man}} \cap C_{\operatorname{man}}\right)=\phi=\frac{\gamma \cdot \varphi}{\gamma+\varphi}$ & $\gamma$ e $\varphi . \phi>0$ \\
\hline
\end{tabular}

Fonte: Lima et al. (2016).

Figura 2 - Índices de Análise de Sensibilidade

A Metodologia Multi-Índice Ampliada (MMIA) foi implementada no Sistema de Análise de Viabilidade Econômica de Projetos de Investimento, denominado $\$ \wedge \bigvee € \pi$. Por recomendação de Lima (2016), o ponto de partida deve ser a construção do diagrama do fluxo de caixa, seguido da seleção e preenchimento do input aplicativo $\$ \wedge \vee € \pi$. Por fim, esse autor recomenda a elaboração de um relatório com os resultados obtidos e a emissão de um parecer conclusivo sobre a viabilidade econômica da aquisição do equipamento.

$\mathrm{Na}$ Figura 3, pode ser observado o modelo de metodologia utilizado. 
- Levantamento do investimento inicial via orçamento;

Etapa 1

- Estimativas, de forma comparativa, dos custos de produção dos processos tais como: Mão de obra; Energia elétrica;

Etapa 2 Manutenção; Consumíveis;

- Aplicação da MMIA sobre a abordagem determinística via

Etapa 3 $\$ \Lambda \vee € \pi ;$

- Análise dos resultados e emissão do parecer conclusivo sobre a Etapa 4 viabilidade econômica do projeto de investimento.

Fonte: Autor (2017).

Figura 3 - Metodologia utilizada 


\section{APRESENTAÇÃO E DISCUSSÃO DOS RESULTADOS}

\subsection{Caracterização da Empresa e do Projeto de Investimento}

O presente estudo de caso foi baseado em dados coletados junto a uma empresa do setor metal mecânico, fundada em agosto de 2001. A fundição, localizada no Sudoeste do estado do Paraná, atende aos mercados nacional e internacional, fornecendo peças fundidas, principalmente para os setores agrícola, automotivo, civil e doméstico, com uma produção mensal de cerca de 250 toneladas.

Desde o início das atividades em 2001, umas das etapas do processo de acabamento de chapas de fogão da lenha, principal produto da linha doméstica, é realizado com um operador que manuseia a peça fornecendo o acabamento de sua lateral. Tal acabamento, além da baixa produtividade, apresenta uma instabilidade dimensional devido ao esforço realizado pelo operador ser instável. Além disso, com o aquecimento do mercado, o aumento da demanda fez com que surgisse a necessidade de ampliar a capacidade do setor. Dessa forma, surgiu a necessidade de analisar economicamente a possibilidade de adicionar mais uma máquina semelhante às existentes ou investir em um sistema automatizado para realizar tal processo. Para fins de análise manteve-se fixa a capacidade de produção, tanto para o processo atual, quanto para o processo futuro. Dessa forma é possível estimar a variação de custos entre o processo atual e futuro.

\subsection{Análise Econômica do Projeto de Investimento Proposto}

\subsubsection{Investimento}

No sistema atual, a empresa trabalhando 8 horas e 40 minutos por dia, tem uma capacidade média mensal de produção de 7.568 chapas ou 344 chapas por dia. O novo processo proposto tem uma expectativa de produção do mesmo número de chapas por mês, trabalhando o mesmo número de horas mensais, porém com apenas um colaborador, ao invés de cinco colaboradores como é necessário no sistema atual. 
O investimento total estimado para a implantação do novo processo de acabamento é de $\mathrm{R} \$ 247.000,00$ sendo analisado a modalidade de execução através de recursos próprios.

\subsection{Custos de Produção dos Processos}

Nesta etapa do projeto de investimento serão coletados os custos de produção envolvidos, tanto para o processo atual quanto para o novo processo, a fim de obter a diferença de custos resultante.

\subsubsection{Mão de obra (MDO)}

Atualmente, devido à capacidade de produção do processo instalado, é possível produzir em torno de 344 chapas em horário comercial ( 8 h e 40 min por dia), sendo esperada a mesma produção com o novo processo. Porém, um fator importante é que no processo atual são necessários 5 colaboradores, enquanto no processo proposto seria necessário apenas um colaborador para o processo. A Tabela 1 apresenta uma comparação entre os dois processos, no que compete aos custos de mão de obra. Tem-se que o custo da hora trabalhada em horário comercial é de $R \$ 11,12$, já incluindo todos os encargos trabalhistas.

Tabela 1 - Comparação de custo de MDO para os processos

\begin{tabular}{lrr}
\hline \multicolumn{1}{c}{ Item } & Processo Atual & Novo Processo \\
\hline Total de Horas Diárias & $8 \mathrm{~h} \mathrm{e} 40 \mathrm{~min}$ & 8 h e 40 min \\
Número de Colaboradores & 5 & 1 \\
Custo MDO Mensal por Colaborador & $\mathrm{R} \$ 2.152,26$ & $\mathrm{R} \$ 2.152,26$ \\
Custo MDO Mensal Total & $\mathbf{R} \mathbf{1 0 . 7 6 1 , 3 0}$ & $\mathbf{R} \mathbf{2 . 1 5 2 , 2 6}$ \\
\hline
\end{tabular}

Fonte: Elaborada pelo autor com base nos dados de pesquisa (2017)

\subsubsection{Energia elétrica}

Para o levantamento deste item foi levada em conta a potência necessária em motores e equipamentos elétricos utilizada para o processo atual, bem como para o novo processo em avaliação, a fim de comparar o custo mensal com energia elétrica nos dois processos, o qual é apresentado na Tabela 2. Cada kWh consumido tem um custo médio de $\mathrm{R} \$ 0,23$ para a empresa. 
Tabela 2 - Comparação de custo de energia elétrica para os processos

\begin{tabular}{lrr}
\hline \multicolumn{1}{c}{ Item } & Processo Atual & \multicolumn{1}{c}{ Novo Processo } \\
\hline Total de Horas Diárias & $8 \mathrm{~h} \mathrm{40 \textrm {min }}$ & $8 \mathrm{~h} \mathrm{40} \mathrm{\operatorname {min }}$ \\
Potência instalada & $18,4 \mathrm{~kW}$ & $9,6 \mathrm{~kW}$ \\
Consumo Diário & $161,7 \mathrm{~kW}$ & $84,084 \mathrm{~kW}$ \\
Custo de Energia Mensal & $\mathbf{R} \$ \mathbf{8 3 1 , 8 4}$ & $\mathbf{R} \$ \mathbf{4 3 2 , 5 6}$ \\
\hline
\end{tabular}

Fonte: Elaborada pelo autor com base nos dados de pesquisa (2017)

\subsubsection{Manutenção}

Tanto para o novo processo quanto para o processo já instalado, existem alguns custos de manutenção devido a desgastes de processo, considerados mais impactantes. Esses valores são apresentados na Tabela 3.

Tabela 3 - Comparação de custo de manutenção para os processos

\begin{tabular}{lrr}
\hline \multicolumn{1}{c}{ Item } & Processo Atual & Novo Processo \\
\hline Custo de Manutenção Mensal & $\mathrm{R} \$ 400,00$ & $\mathrm{R} \$ 50,00$ \\
\hline Fonte: Elaborada pelo autor com base nos dados de pesquisa (2017) &
\end{tabular}

\subsubsection{Consumíveis}

Para o processo de acabamento das chapas é necessária a utilização de rebolos, os quais tem uma vida útil limitada. $O$ processo atual utiliza um rebolo que tem um custo de $R \$ 700,00$ e uma vida útil de 340 chapas produzidas, enquanto o processo proposto utilizaria um rebolo o qual tem um custo de $R \$ 9.000,00$ e uma vida útil de 3.400 chapas produzidas.

Com o objetivo de comparar os custos mensais de consumíveis, foi estimada uma quantidade de 344 chapas diárias para o cálculo. Tal comparação pode ser vista na Tabela 4.

Tabela 4 - Comparação de custo do rebolo utilizado em ambos os processos

\begin{tabular}{|c|c|c|c|c|}
\hline Item & \multicolumn{2}{|c|}{ Processo Atual } & \multicolumn{2}{|c|}{ Novo Processo } \\
\hline Custo do Rebolo & $\mathrm{R} \$$ & 700,00 & $\mathrm{R} \$$ & $9.000,00$ \\
\hline Qtde peças produzidas / Rebolo & & 340 & & 3.400 \\
\hline Custo do Rebolo / Peça Produzida & & $\mathbf{R} \$ 2,06$ & & $\mathbf{R} \$ 2,65$ \\
\hline Custo do Rebolo Mensal & & $R \$ 15.581,18$ & & $R \$ 20.032,94$ \\
\hline
\end{tabular}

Fonte: Elaborada pelo autor com base nos dados de pesquisa (2017) 


\subsection{Comparativo entre os dois processos}

Após a obtenção dos dados explanados anteriormente, os mesmos foram reunidos na Tabela 5, a fim de comparar o custo envolvido no acabamento de chapas no processo atual com o processo proposto nesse trabalho. Para a estimativa do custo, foi considerada uma produção constante de 344 chapas diárias, sendo esta a produção esperada para os dois sistemas.

A diferença de custo apresentada na Tabela 5 pode ser percebida como 0 valor que deixará de ser gasto para a etapa de acabamento de cada chapa de fogão. Para chegar aos números de produção utilizados, foi consultado o diretor industrial da empresa proponente do projeto, por meio de entrevistas individuais semiestruturadas.

Tabela 5 - Comparativo de custos de acabamento entre os dois processos

\begin{tabular}{|c|c|c|}
\hline Custos mensais & Processo Atual & Novo Processo \\
\hline Mão de Obra (MDO) & $\mathrm{R} \$ 10.761,30$ & $\mathrm{R} \$ 2.152,26$ \\
\hline Energia Elétrica & $\mathrm{R} \$ 831,84$ & $\mathrm{R} \$ 432,56$ \\
\hline Insumos (Rebolo) & $\mathrm{R} \$ 15.581,18$ & $\mathrm{R} \$ 20.032,94$ \\
\hline Manutenção & $R \$ 400,00$ & $R \$ 50,00$ \\
\hline Custo Mensal & $\mathrm{R} \$ 27.574,31$ & $\mathrm{R} \$ 22.667,76$ \\
\hline Produção diária & \multicolumn{2}{|c|}{344} \\
\hline Produção Mensal & \multicolumn{2}{|c|}{7.568} \\
\hline Custo por Chapa & $R \$ 3,64$ & $R \$ 3,00$ \\
\hline Diferença de Custo por Peça & & $R \$ 0,65$ \\
\hline
\end{tabular}

Fonte: Elaborada pelo autor com base nos dados de pesquisa (2017)

\subsection{Estudo de Viabilidade Econômica do Projeto de Investimento}

\subsubsection{Inserção dos dados no Aplicativo Web $\$ \Lambda V € \pi$ sem impostos}

Após o levantamento dos dados apresentados na seção anterior, foi utilizado o aplicativo Web $\$ \Lambda \bigvee € \pi$ para geração dos indicadores e gráficos para posterior análise. O montante aplicado no PI é de $R \$ 247.000,00$, para a aquisição e instalação dos equipamentos necessários para o funcionamento do novo processo.

Na Figura 4 estão detalhados os dados do PI. Para este PI foi estimado um horizonte de planejamento $(\mathrm{N})$ de 10 anos (120 meses), considerando o Valor Residual (VR) como nulo, pois não há expectativa de revenda dos equipamentos ao final desse horizonte. 


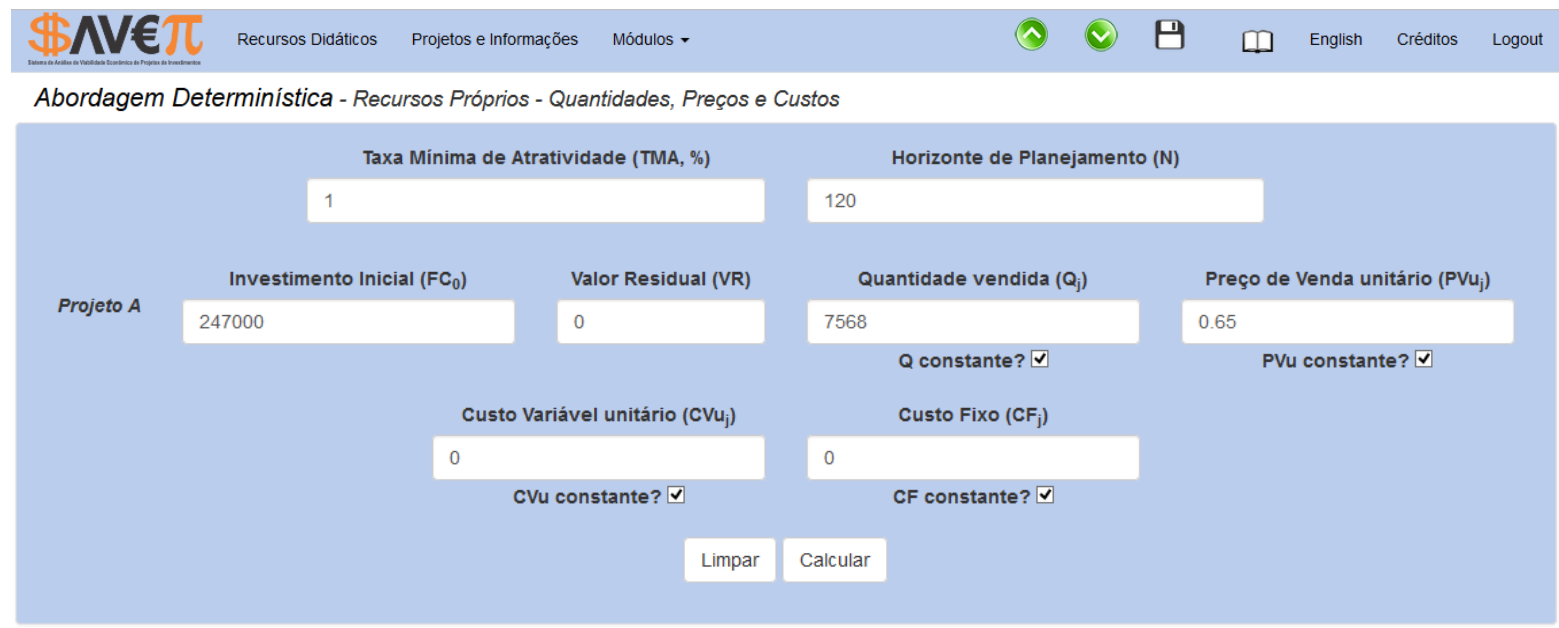

Fonte: Elaborada pelo autor no Aplicativo Web $\$ \Lambda V € \pi$ (2017)

Figura 4 - Input dos dados no Aplicativo Web $\$ \Lambda V € \pi$

\subsubsection{Inserção dos dados no Aplicativo Web $\$ \Lambda V € \pi$ com impostos}

Após consulta ao setor financeiro da empresa, foi considerado o Imposto de Renda (IR) como sendo optante do lucro real cujo valor é $15 \%$ e a Contribuição Social Sobre o Lucro Líquido (CSLL) de 9\%. Utilizando o aplicativo Web $\$ \Lambda \mathrm{V} € \pi$, o input dos dados pode ser observado na Figura 5.

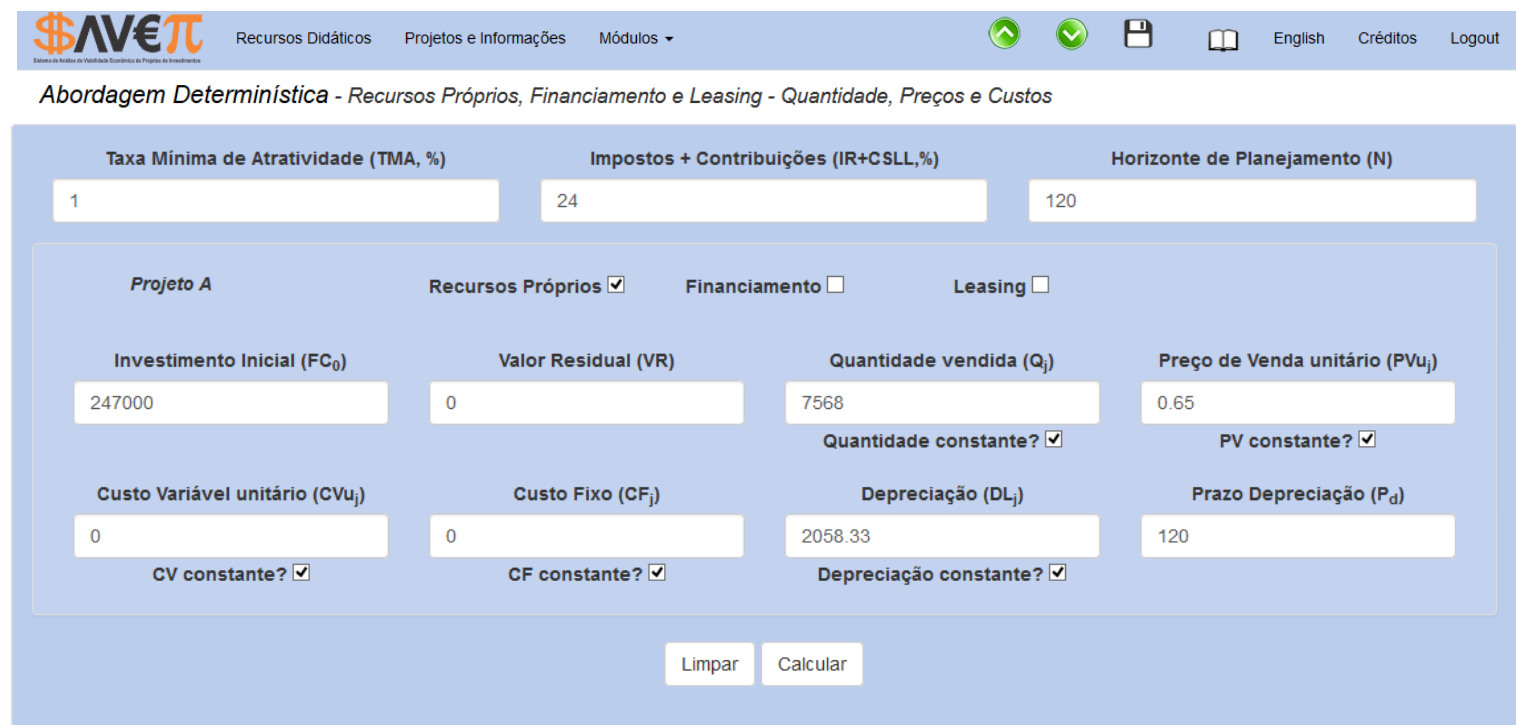

Fonte: Elaborada pelo autor no $\$ \Lambda \bigvee € \pi(2017)$

Figura 5 - Input dos dados no $\$ \Lambda V € \pi$, com impostos 


\subsubsection{Principais resultados obtidos}

Na Figura 6, a qual foi gerada automaticamente pelo Aplicativo Web $\$ \Lambda \bigvee € \pi$ após a inserção dos dados no input, são mostrados o comparativo dos resultados dos indicadores econômicos com e sem os impostos. Para ambas as situações são gerados os seguintes indicadores: Dimensão retorno (VP, VPL, VPLA, IBC, ROIA, índice ROIA/TMA e ROI); Dimensão riscos (TIR, TMA/TIR, Payback e Payback/N); Dimensão sensibilidade (Variação da TMA, variação do FCo, variação de Qj e variação de PVuj, os quais são correspondentes à variação da TMA, do investimento inicial, da quantidade vendida e do preço unitário, respectivamente).

\begin{tabular}{|c|c|c|c|c|}
\hline Dimensão & Indicador & $\begin{array}{c}\text { Resultado Esperado (se } \\
\text { viável) }\end{array}$ & $\begin{array}{l}\text { Valor esperado } \\
\text { sem impostos }\end{array}$ & $\begin{array}{l}\text { Valor esperado } \\
\text { com impostos }\end{array}$ \\
\hline \multirow{6}{*}{ Retorno } & VP $(R \$)$ & $\mathrm{VP} \geq\left|\mathrm{FC}_{0}\right|$ & $342.870,81$ & $295.013,82$ \\
\hline & VPL $(R \$)$ & $\mathrm{VPL} \geq 0$ & $95.870,81$ & $48.013,82$ \\
\hline & VPLA (R\$) & VPLA $\geq 0$ & $1.375,47$ & 688,86 \\
\hline & IBC & $\mathrm{IBC}_{1} \geq 1$ & 1,3881 & 1,1944 \\
\hline & ROIA (\%) & ROIA $\geq 0$ & 0,27 & 0,15 \\
\hline & Índice ROIA/TMA (\%) & Índice ROIA/TMA $\geq 0$ & 27,37 & 14,81 \\
\hline \multirow{4}{*}{ Riscos } & Payback (meses) & Payback $\leq \mathrm{N}$ & 71 & 89 \\
\hline & TIR (\%) (ao mês) & TIR $\geq$ TMA & 1,74 & 1,38 \\
\hline & Índice Payback/N (\%) & Índice Payback/N $\leq 100 \%$ & 59,17 & 74,17 \\
\hline & Índice TMA/TIR (\%) & Índice TMA/TIR $\leq 100 \%$ & 57,46 & 72,23 \\
\hline \multirow{4}{*}{$\begin{array}{l}\text { Limites de } \\
\text { Elasticidade }\end{array}$} & $\Delta \%$ TMA & Quanto > a tolerância Melhor & 74,04 & 38,45 \\
\hline & $\Delta \% \mathrm{FC}_{0}$ & Quanto > a tolerância Melhor & 38,81 & 19,44 \\
\hline & $\Delta \% \mathrm{Q}_{\mathrm{j}}$ & Quanto > a tolerância Melhor & 27,96 & 18,07 \\
\hline & $\Delta \% \mathrm{PVu}_{\mathrm{j}}$ & Quanto > a tolerância Melhor & 27,96 & 18,07 \\
\hline
\end{tabular}

Fonte: Elaborada pelo autor no Aplicativo Web $\$ \Lambda \bigvee € \pi n 2017$

Figura 6 - Comparativo dos Indicadores da Metodologia Multi-índice Ampliada - MMIA

\subsection{Análise dos Indicadores de Retorno}

É esperado que a empresa recupere o investimento realizado caso esse PI seja executado, dessa forma, substituir o processo atual pelo novo processo, gerando um retorno maior do que se o montante for aplicado no mercado a uma taxa de 1,0\% ao mês, para ambos os casos analisados. Na Figura 7, pode ser observado os indicadores de retorno. 


\begin{tabular}{|l|l|l|c|c|}
\hline \multirow{2}{*}{ Dimensão } & \multicolumn{1}{|c|}{ Indicador } & \multicolumn{1}{|c|}{$\begin{array}{c}\text { Resultado Esperado (se } \\
\text { viável) }\end{array}$} & $\begin{array}{c}\text { Valor esperado } \\
\text { sem impostos }\end{array}$ & $\begin{array}{c}\text { Valor esperado } \\
\text { com impostos }\end{array}$ \\
\hline \multirow{5}{*}{ Retorno } & $\mathrm{VP}(\mathrm{R} \$)$ & $\mathrm{VP} \geq \mid \mathrm{FC}$ 0 & $342.870,81$ & $295.013,82$ \\
\cline { 2 - 5 } & $\mathrm{VPL}(\mathrm{R} \$)$ & $\mathrm{VPL} \geq 0$ & $95.870,81$ & $48.013,82$ \\
\cline { 2 - 5 } & $\mathrm{VPLA}(\mathrm{R} \$)$ & $\mathrm{VPLA} \geq 0$ & $1.375,47$ & 688,86 \\
\cline { 2 - 5 } & $\mathrm{IBC}$ & $\mathrm{IBC} \geq 1$ & 1,3881 & 1,1944 \\
\cline { 2 - 5 } & $\mathrm{ROIA}(\%)$ & $\mathrm{ROIA} \geq 0$ & 0,27 & 0,15 \\
\cline { 2 - 5 } & Índice ROIA/TMA (\%) & Índice ROIA/TMA $\geq 0$ & 27,37 & 14,81 \\
\hline
\end{tabular}

Fonte: Elaborada pelo autor no $\$ \Lambda \bigvee € \pi$ (2017)

Figura 7 - Comparativo dos Indicadores de retorno utilizando a MMIA

O indicador Valor Presente Líquido (VPL), mostrado na Figura 7, pode ser entendido como o retorno que se obteve além do que seria obtido se o mesmo investimento inicial (FCo) tivesse sido aplicado em um investimento o qual rendesse o mesmo que a TMA adotada pela empresa. Segundo Souza e Clemente (2009), não é recomendado tomar uma decisão baseada apenas neste indicador, porém como o mesmo teve resultado positivo, o $\mathrm{PI}$ continuará a ser analisado.

O indicador Valor Presente Líquido Anualizado (VPLA) é útil, pois o mesmo representa o Fluxo de Caixa (FC) descapitalizado para o período presente é distribuído por unidade de tempo, isto é, quanto o PI renderia mensalmente, sendo este valor adicional ao que renderia caso $\mathrm{FC}_{0}$ fosse investido com rendimento igual à TMA utilizada. Como o VPLA resultou em $\mathrm{R} \$ 1.375,47$ sem considerar os impostos e $\mathrm{R} \$ 688,86$ considerando os impostos sobre o lucro, sendo para ambos os casos, resultou um valor positivo, o $\mathrm{PI}$ continuará a ser analisado.

O Índice Benefício/Custo (IBC) resultou no valor de 1,3881 sem considerar impostos e em 1,1944 considerando impostos. Tal índice representa que a cada $\mathrm{R} \$$ 1,00 investido, ocorrerá um retorno de $R \$ 1,39$ e $R \$ 1,19$ respectivamente ao final do horizonte de planejamento, já subtraído o retorno que se obteria caso o montante inicial fosse aplicado com uma taxa equivalente à TMA.

O Retorno Adicional sobre o Investimento (ROIA) sem impostos foi de 0,27\% além da TMA utilizada e com impostos foi de 0,15\%. A fim de auxiliar na decisão sobre o $\mathrm{PI}$ pode ser utilizado o índice ROIA/TMA, pois este quantifica quanto 0 investimento poderá lucrar além da TMA. O valor atingido para o índice ROIA/TMA foi de $27,37 \%$ sem imposto e $14,81 \%$ com impostos.

Após a análise dos principais índices de retorno, pode-se concluir que o projeto de investimento proposto tem um retorno esperado do nível Baixo-Médio sem 
considerar impostos, bem como um retorno baixo considerando impostos, segundo escala proposta por Lima (2017), porém ainda positivo, sendo necessário avaliar os indicadores de riscos e de elasticidade com o intuito de obter um parecer final com mais solidez.

\subsection{Análise dos Indicadores de Risco}

Na Figura 8, pode ser observado o comparativo entre os indicadores de risco:

\begin{tabular}{|c|l|l|c|c|}
\hline \multirow{2}{*}{ Dimensão } & \multicolumn{1}{|c|}{ Indicador } & \multicolumn{1}{|c|}{$\begin{array}{c}\text { Resultado Esperado (se } \\
\text { viável) }\end{array}$} & $\begin{array}{c}\text { Valor esperado } \\
\text { sem impostos }\end{array}$ & $\begin{array}{c}\text { Valor esperado } \\
\text { com impostos }\end{array}$ \\
\hline \multirow{4}{*}{ Riscos } & Payback (meses) & Payback $\leq \mathrm{N}$ & 71 & 89 \\
\cline { 2 - 5 } & TIR (\%) (ao mês) & TIR $\geq$ TMA & 1,74 & 1,38 \\
\cline { 2 - 5 } & Índice Payback/N (\%) & Índice Payback/N $\leq 100 \%$ & 59,17 & 74,17 \\
\cline { 2 - 5 } & Índice TMA/TIR (\%) & Índice TMA/TIR $\leq 100 \%$ & 57,46 & 72,23 \\
\hline
\end{tabular}

Fonte: Elaborada pelo autor no $\$ \Lambda \bigvee € \pi$ (2017)

Figura 8 - Comparativo dos Indicadores de risco utilizando a MMIA

A Taxa Interna de Retorno (TIR), segundo Souza e Clemente (2009) pode ser explicada como a taxa que define um limite máximo para a variação da TMA proposta. Este indicador teve como resultado o valor de 1,74\% sem considerar impostos, bem como $1,38 \%$ considerando os impostos sobre o lucro líquido. Outro indicador interessante para auxiliar na tomada de decisão é o índice TMA/TIR, o qual pode ser interpretado como o risco decorrente ao investir no projeto (SOUZA e CLEMENTE, 2009), sendo assim, quanto menor o índice obtido, mais seguro é de se investir no PI em questão. Foi obtido o valor de 57,46\% considerado médio risco para o caso sem impostos e $72,23 \%$ com impostos, o qual é considerado médio-alto risco segundo escala proposta por Lima (2017).

Outro indicador de risco que deve ser levado em conta é o Payback, ou seja, o período de tempo necessário para a recuperação do montante inicialmente investido. O projeto proposto apresentou um Payback de 71 meses sem impostos e 89 meses com impostos, em horizonte de 120 meses. Um indicador relacionado a este é o índice Payback/N, o qual compara o Payback com o horizonte de planejamento (N) do projeto. Quanto menor este índice, menor o risco do PI não recuperar o investimento inicial. Foi obtido um Payback/N no valor de 59,17\% considerado um risco Médio segundo escala proposta por Lima (2017) sem 
considerar os impostos e $74,17 \%$ considerando impostos, sendo este risco considerado como Médio-Alto segundo escala proposta por Lima (2017).

\subsection{Análise dos Limites de Elasticidade e Comparativo entre Retorno e Riscos}

Na Figura 9, pode ser observado o comparativo entre os indicadores de risco:

\begin{tabular}{|c|l|l|c|c|}
\hline \multirow{2}{*}{ Dimensão } & \multicolumn{1}{|c|}{ Indicador } & \multicolumn{1}{c|}{$\begin{array}{c}\text { Resultado Esperado (se } \\
\text { viável) }\end{array}$} & $\begin{array}{c}\text { Valor esperado } \\
\text { sem impostos }\end{array}$ & $\begin{array}{c}\text { Valor esperado } \\
\text { com impostos }\end{array}$ \\
\hline \multirow{3}{*}{$\begin{array}{c}\text { Limites de } \\
\text { Elasticidade }\end{array}$} & $\Delta \% \mathrm{TMA}$ & Quanto > a tolerância Melhor & 74,04 & 38,45 \\
\cline { 2 - 5 } & $\Delta \% \mathrm{FC}_{0}$ & Quanto > a tolerância Melhor & 38,81 & 19,44 \\
\cline { 2 - 5 } & $\Delta \% \mathrm{Q}_{\mathrm{j}}$ & Quanto > a tolerância Melhor & 27,96 & 18,07 \\
\cline { 2 - 5 } & $\Delta \% \mathrm{PV}_{\mathrm{j}}$ & Quanto > a tolerância Melhor & 27,96 & 18,07 \\
\hline
\end{tabular}

Fonte: Elaborada pelo autor no $\$ \Lambda \bigvee € \pi$ (2017)

Figura 9 - Comparativo dos Indicadores de risco utilizando a MMIA

Os indicadores de retorno, risco e limites de elasticidade podem ser avaliados segundo escala proposta por Lima (2017), os quais podem ser vistos nas Figuras 10 e 11 .

\begin{tabular}{|c|c|c|c|c|c|c|c|c|}
\hline CATEGORIA & INDICE & Observação & BAIXO & BAIXO-MÉDIO & MÉDIO & MÉDIO-ALTO & ALTO & Observação \\
\hline RETORNO & ROIATMA & - & & 27,37 & & & & - \\
\hline \multirow{2}{*}{ RISCOS } & Payback/N ${ }^{1}$ & - & & & 59,17 & & & - \\
\hline & TMA $_{T} \mathrm{IR}^{2}$ & - & & & 57,46 & & & - \\
\hline \multirow{4}{*}{ LIMITES DE ELASTICIDADE } & $\triangle \%$ TMA & - & & & & 74,04 & & - \\
\hline & $\Delta \% \mathrm{FC}_{0}$ & & & 38,81 & & & & \\
\hline & $\Delta \% Q_{j}$ & & & 27,96 & & & & - \\
\hline & $\Delta \% \mathrm{PVu}_{\mathrm{j}}$ & & & 27,96 & & & & \\
\hline \multicolumn{2}{|l|}{ Escala proposta } & $<0 \%$ & $0 \%$ a $20 \%$ & $20 \%$ a $40 \%$ & $40 \%$ a $60 \%$ & $60 \%$ a $80 \%$ & $80 \%$ a $100 \%$ & $>100 \%$ \\
\hline
\end{tabular}

Fonte: Elaborada pelo autor no $\$ \Lambda \mathrm{V} € \pi$ (2017).

Figura 10 - Confronto retorno esperado versus riscos estimados sem impostos (LIMA, 2017)

\begin{tabular}{|c|c|c|c|c|c|c|c|c|}
\hline CATEGORIA & ÍNDICE & OBS & BAIXO & $\begin{array}{l}\text { BAIXO- } \\
\text { MÉDIO }\end{array}$ & MÉDIO & $\begin{array}{c}\text { MÉDIO- } \\
\text { ALTOO }\end{array}$ & ALTO & OBS \\
\hline RETORNO & ROIA/TMA & - & 14,81 & & & & & - \\
\hline \multirow{2}{*}{ RISCOS } & Payback/N ${ }^{1}$ & - & & & & 74,17 & & - \\
\hline & TMA/TIR ${ }^{2}$ & - & & & & 72,23 & & - \\
\hline \multirow{3}{*}{ LIMITES DE ELASTICIDADE } & $\triangle \%$ TMA & - & & 38,45 & & & & - \\
\hline & $\Delta \% \mathrm{FC}_{0}$ & - & 19,44 & & & & & - \\
\hline & $\Delta \% \mathrm{FC}_{\mathrm{j}}$ & - & 16,28 & & & & & $=$ \\
\hline
\end{tabular}

Fonte: Elaborada pelo autor no $\$ \Lambda \mathrm{V} € \pi$ (2017).

Figura 11 - Confronto retorno esperado versus riscos estimados com impostos (LIMA, 2017)

O indicador de variação da TMA denota que para inviabilizar economicamente o PI, a TMA teria que aumentar em 74,04\% para o caso de não considerar impostos, bem como $38,45 \%$ considerando os impostos. Já o indicador de variação da FCo 
mostra que o investimento inicial poderia ter um aumento de $38,81 \%$, ou seja, ter um valor limite de $R \$ 342.860,70$, sendo esse limite de variação considerados baixomédio, segundo escala proposta por Lima (2017), enquanto que para o caso com impostos, esse índice poderia ter um aumento de até 19,44\%, tendo como valor limite $R \$ 295.016,80$ e se enquadra em um baixo limite de elasticidade para tal indicador, segundo escala proposta por Lima (2017).

Para o indicador de variação de quantidade esperada de vendas (Qj), é admitida uma diminuição nas vendas na ordem de 27,96\% (mínimo 5.452 unidades) e 18,07\% (mínimo 6.200 unidades) para os casos sem e com impostos, respectivamente, sendo este limite de elasticidade considerado baixo-médio.

O índice de variação de PVuj, neste caso representa que a diferença entre o custo de produção do processo atual para o processo futuro admite uma diminuição na diferença dos custos de até 27,96, assim a diferença entre os custos do processo atual e futuro pode reduzir para até $R \$ 0,47$ para o caso sem considerar impostos. Considerando os impostos é admitido uma redução de até 18,07\%, dessa forma, o menor valor da diferença entre os custos dos processos admissível é de $R \$ 0,53$. 


\section{CONSIDERAÇÕES FINAIS}

O objetivo proposto nesse estudo foi analisar economicamente a automatização do processo por meio da aquisição de um novo equipamento. Os resultados apresentados mostram a quantidade de informações geradas com a aplicação da MMIA.

Para o projeto em estudo foi avaliado as dimensões retorno a qual apresentou uma expectativa de ganho financeiro de $27,37 \%$ além da TMA definida pela empresa. Diante dos dados mensurados, ao combinar os riscos medidos pelos índices Payback/N e TMA/TIR, o PI se enquadra na categoria de risco com grau médio com valor percentual de $58,3 \%$. Contudo, para melhorar a percepção dos riscos desse investimento, considerando a análise de sensibilidade nos principais parâmetros intervenientes no desempenho econômico do $\mathrm{PI}$ em análise. O PI apresentou baixa-média sensibilidade.

Conclui-se com os dados coletados e a realização da simulação para os casos sem e com imposto, que é recomendado a aquisição do equipamento analisado.

Como projeto futuro propõe-se que a companhia realize estudo de viabilidade econômica utilizando a Análise de Cenários como Artuzo et al. utilizaram ou Simulação de Monte Carlo, pois o custo unitário de rebolo para o equipamento em análise pode apresentar elevadas variações devido ao valor de produtividade, ser especificado pelo fornecedor do rebolo e pode variar de acordo com o acabamento superficial das peças bem como dureza das mesmas. Também pode ser realizada uma pesquisa para simular o investimento com recursos adquiridos por meio de financiamento. 


\section{REFERÊNCIAS}

ARTUZO, R.; ULSENHEIMER, L.; DAROS, R.; LIMA, J. D. DE; SETTI, D. Estudo de viabilidade econômica da alteração do processo de moldagem de chapas de fogão a lenha. VI Congresso Brasileiro de Engenharia de Produção. Ponta Grossa, PR, Brasil, 2016.

BREALEY, R..A.; MYERS, S. C. Princípios de Finanças Empresariais. Tradução H. Caldeira Menezes e J.C. Rodrigues da Costa. 3. ed. Portugal: McGraw-Hill de Portugal, 1992.

BUARQUE, C.R.C. Avaliação Econômica de Projetos. Rio de Janeiro: Elsevier, 1989.

CASAROTTO FILHO, N.; KOPITTKE, B.H. Análise de Investimentos: Matemática Financeira, Engenharia Econômica, Tomada de Decisão, Estratégia Empresarial. 11. ed. São Paulo: Atlas, 2010.

FERRO, W. A.; LIMA J. D. DE; SETTI, D. Viabilidade econômica da aquisição de uma linha de pintura associada ao tratamento de superfície de metais com tecnologia nanocerâmica. Revista Espacios. v.37, n.26. p.19. 2016.

HARZER, J. H.; SOUZA A.; DUCLÓS L.C.. Método de Monte Carlo Aplicado à Análise de Projeto: Estudo de Investimento em um Empreendimento Hoteleiro. 2012.

HARZER, J. H.; SOUZA A.; SILVA, W. V. da; CRUZ, J. A. W. C.. Abordagem Probabilística do Indicador TMA/TIR Para Avaliação do Risco Financeiro em Projetos de Investimentos. XXI Congresso Brasileiro de Custos - Natal, RN, Brasil, 2014.

KASSAI, J. R.; KASSAI, S.; SANTOS, A. dos; ASSAF NETO, A. Retorno de Investimento: Abordagem Matemática e Contábil do Lucro Empresarial. São Paulo: Atlas 2000.

LIMA, J.D. de; TRENTIN, M.G.; OLIVEIRA, G.A.; BATISTUS, D.R.; SETTI, D. A systematic approach for the analysis of the economic viability of investment projects. Int. J. Engineering Management and Economics. v.5, n.1/2. p.19-34. 2015.

LIMA, J.D. de. Manual de Análise da Viabilidade Econômica de Projetos de Investimentos (MAVEPI): abordagem determinística. Notas de aula - textos para discussão. Universidade Tecnológica Federal do Paraná (UTFPR - Câmpus Pato Branco). Programa de Pós-graduação em Engenharia de Produção e Sistemas (PPGEPS). 2016. Disponível em: http://pb.utfpr.edu.br/savepi/materialDeApoio.php.

LIMA, J.D. de, ALBANO, J.C. da S., OLIVEIRA, G.A., TRENTIN, M.G., BATISTIUS, D.R. Estudo de viabilidade econômica da expansão e automatização do setor de embalagem em agroindústria avícola. Custos e agronegócio online v. 12, n. 1 - Jan/Mar - 2016. 
NOGUEIRA, E. Análise de Investimentos. In. BATALHA, M.O. (coord.). Gestão Agroindustrial. 5. ed. v.2. São Paulo: Atlas, 2009. p. 205-266.

RASOTO, A.; GNOATTO, A.A.; OLIVEIRA, A.G. de; ROSA, C.F. da; ISHIKAWA, G.; CARVALHO, H.A. de; LIMA, I.A. de; LIMA, J.D. de; TRENTIN; M.G.; V.I. Gestão Financeira: enfoque em inovação. 1. ed. Curitiba: Aymará, 2012. v. 6. (série UTFinova).

ROSA, F. do E. S.. Planejando as Finanças de Sua Empresa. Monografia, Universidade Cândido Mendes. Rio de Janeiro, 2010.

ROZENFELD, H.; FORCELLINI, F.A.; AMARAL, D.C.; TOLEDO. J.C.; SILVA, S.L.; ALLIPRANDINI, D.H.; SCALICE, R.K. Gestão de Desenvolvimento de Produtos: Uma referência para a melhoria do processo. 1. ed. São Paulo: Saraiva, 2006.

$\$ \wedge \vee € \pi$. Sistema de Análise da Viabilidade Econômica de Projetos de Investimentos. Disponível em: <http://pb.utfpr.edu.br/savepi/cadastrar.php/>. Acesso em: Fev. 2017.

SOUZA, A.; CLEMENTE, A. Decisões Financeiras e Análises de Investimentos: Conceitos, técnicas e aplicações. 6. ed. São Paulo: Atlas, 2008.

STRACHOSKI, P. Análise de Viabilidade Econômica de um Projeto de Investimento em uma Indústria de Artefatos de Cimentos. Trabalho de Conclusão de Curso, Criciúma, SC, 2011. 